\title{
GSE MOVES TO OPEN ACCESS PUBLISHING
}

We are pleased to announce that from 1st January 2009 GSE will become the first "Open Access" (OA) journal dedicated to original research on genetics and selection in farm and experimental animals. With this editorial, we would like to introduce $\mathrm{OA}$ and provide some reasons leading to this decision.

For the past two years, there has been much debate about OA as a viable economic model for publishing science. We feel that the current subscriptionbased system restricts availability and circulation of scientific information. INRA, a French public research body that owns GSE, adheres to the Open Access principles and has signed the "2003 Berlin declaration on Open Access to knowledge in the Sciences and Humanities". Although the financial situation of GSE in 2007 is stable and subscriptions come from over 45 countries, we feel that the number of subscriptions is a strong limitation on the journal's readership and recognition.

In recent years, GSE has gradually prepared for this conversion i.e. free access to its articles 12 months after publication, a low fee Open Access option and, since September 2007, free access to the entire collection of back issues of GSE since 1969. The efficiency of this policy is illustrated by the increasing number of articles downloaded, around 160000 in 2007.

Open Access is defined as: free, immediate, permanent and full-text online access, for any user, to peer reviewed research. OA articles are archived in freely accessible international repositories including PubMed Central. Authors retain copyright and agree that anyone is free to copy, distribute or display the work provided that the original authors are given credit. The economic basis is that authors are charged a fee to have their work published instead of the readers to access articles. We believe that this type of publication cost can be met through research grants and represents only a small percentage of research budgets.

The expected advantages of OA are (1) increased visibility and accessibility of both the articles and the journal, (2) user-friendly system, (3) immediate access to accepted articles without waiting for the complete issue, (4) improved communication among researchers around the world, (5) increased citations, (6) increased number of submissions and (7) new services that traditional 
publishing cannot offer i.e. more articles and pages, free colour figures, availability of automatic tools that index and cross-reference articles, possibility to post comments about an article on the journal's website.

GSE will move to OA from January 2009. Due to processing time, all manuscripts submitted since 1st April 2008 and accepted will be published in this new framework.

We believe that this is the right time to carry out such a big change because GSE will be the first OA journal in its field. Nevertheless, the journal GSE will remain the full property of the INRA and there will be no change in the journal's title and scope of interests, in the organisation of the editorial management and in the peer-review process.

To accompany this conversion to OA and to reduce author fees, during 2009 GSE and INRA will act as sponsors and charge authors only 750 euros for each accepted article. This fee is significantly less than the cost price of OA publication and thus represents a reasonable pricing compared to that of other OA journals.

However, this change in economic model from a subscription basis to an author payment system remains a risky venture. Its economic viability is highly dependent on the number of good quality manuscripts submitted in the near future i.e. on the authors joining forces for its development.

Thus we take this opportunity to invite you to contribute papers for OA publication, which will benefit from extended readership and thus an increased number of downloads and citations.

For more details, please consult the journal's website at: http://www. gse-journal.org/ or contact us by email at: helene.hayes@jouy.inra.fr. 